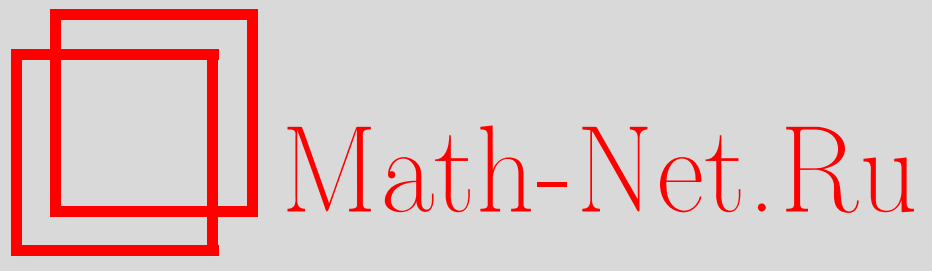

L. Cavalier, Yu. F. Golubev, O. V. Lepskiř, A. Tsybakov, Block thresholding and sharp adaptive estimation in severely ill-posed inverse problems, Teoрия вероятн. и ее примен., 2003, том 48, выпуск $3,534-556$

DOI: https://doi.org/10.4213/tvp269

Использование Общероссийского математического портала MathNet.Ru подразумевает, что вы прочитали и согласны с пользовательским соглашением

http://www.mathnet.ru/rus/agreement

Параметры загрузки:

IP : 54.147 .182 .235

26 апреля 2023 г., 14:50:00

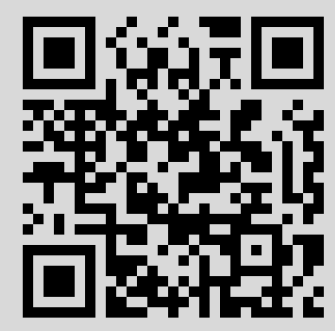


(C) 2003 г. CAVALIER L.*, GOLUBEV Y.*, LEPSKI O.*, TSYBAKOV A.**

\title{
BLOCK THRESHOLDING AND SHARP ADAPTIVE ESTIMATION IN SEVERELY ILL-POSED INVERSE PROBLEMS ${ }^{1)}$
}

\begin{abstract}
Рассматривается задача решения операторного уравнения по зашумленным данным. Предполагается, что $s$-числа оператора экспоненциально убывают и что коэффициенты разложения Фурье решения уравнения сходятся к нулю также с экспоненциальной скоростью. Предлагается оценка решения, основанная на пороговом усечении по скользящим блокам в пространстве коэффициентов Фурье. Показывается, что такой метод оценивания позволяет точно адаптироваться к неизвестной гладкости решения.
\end{abstract}

Ключевые слова и фразы: линейное операторное уравнение, белый гауссовский шум, адаптивное оценивание, пороговое усечение по скользящим блокам.

1. Introduction. The problem of solving linear operator equations from noisy observations has been extensively studied in the literature. Among the first to develop a statistical approach to this problem were Sudakov and Khalfin [17] and Bakushinskii [1]. For a survey of recent results we refer to Mathé and Pereverzev [14], Goldenshluger and Pereverzev [7], Cavalier and Tsybakov [3].

A usual statistical framework in this context is as follows. Let $K: H \rightarrow H$ be a known linear operator on a Hilbert space $H$ with inner product $(\cdot, \cdot)$ and norm $\|\cdot\|$. The problem is to estimate an unknown function $f \in H$ from indirect observations

$$
Y(g)=(K f, g)+\varepsilon \xi(g), \quad g \in H,
$$

where $0<\varepsilon<1$ and $\xi(g)$ is a zero-mean Gaussian random process indexed by $H$ on a probability space $(\Omega, \mathscr{A}, \mathbf{P})$, such that $\mathbf{E}\{\xi(g) \xi(v)\}=(g, v)$ for any $g, v \in H$, where $\mathbf{E}$ is the expectation w.r.t. $\mathbf{P}$. Relation (1) defines a Gaussian white noise model. France.

* CMI, Université Aix-Marseille 1, 39 rue F. Joliot-Curie, F-13453 Marseille Cedex,

** Laboratoire de Probabilités et Modèles Aléatoires, Université Paris 6, 4 pl. Jussieu, BP 188, F-75252 Paris Cedex 05, France. 
Instead of dealing with all the observations $\{Y(g), g \in H\}$, it is usually sufficient to consider a sequence of values $\left\{Y\left(g_{k}\right)\right\}_{k=1}^{\infty}$, for some orthonormal basis $\left\{g_{k}\right\}_{k=1}^{\infty}$. The corresponding random errors $\xi\left(g_{k}\right)=\xi_{k}$ are i.i.d. standard Gaussian random variables.

We assume that the basis $\left\{g_{k}\right\}$ is such that $\left(K f, g_{k}\right)=b_{k} \theta_{k}$, where $b_{k} \neq 0$ are real numbers and $\theta_{k}=\left(f, \varphi_{k}\right)$ are the Fourier coefficients of $f$ w.r.t. some orthonormal basis $\left\{\varphi_{k}\right\}$ (not necessarily $\varphi_{k}=g_{k}$ ). A typical example when it occurs is that the operator $K$ admits a singular value decomposition:

$$
K \varphi_{k}=b_{k} g_{k}, \quad K^{*} g_{k}=b_{k} \varphi_{k},
$$

where $K^{*}$ is the adjoint of $K, b_{k}$ are singular values, $\left\{g_{k}\right\}$ is an orthonormal basis in Range $(K)$ and $\left\{\varphi_{k}\right\}$ is the corresponding orthonormal basis in $H$.

Under these assumptions, one gets a discrete sequence of observations derived from (1):

$$
y_{k}=b_{k} \theta_{k}+\varepsilon \xi_{k}, \quad k=1,2, \ldots,
$$

where $y_{k}=Y\left(g_{k}\right)$ and $\xi_{i}$ are i.i.d. standard Gaussian random variables. The problem of estimating $f$ reduces to estimation the sequence $\left\{\theta_{k}\right\}_{k=1}^{\infty}$ from observations (3). The model (3) also describes other problems such as the estimation of a signal from direct observations with correlated data (see Johnstone [11]).

Let $\hat{\theta}=\left(\hat{\theta}_{1}, \hat{\theta}_{2}, \ldots\right)$ be an estimator of $\theta=\left(\theta_{1}, \theta_{2}, \ldots\right)$ based on the data (3). Then $f$ is estimated by $\hat{f} c=\sum_{k} \hat{\theta}_{k} \varphi_{k}$. The mean integrated squared error of the estimator $\hat{f}$ is

$$
\mathbf{E}_{f}\|\hat{f}-f\|^{2}=\mathbf{E}_{\theta} \sum_{k=1}^{\infty}\left(\hat{\theta}_{k}-\theta_{k}\right)^{2} \stackrel{\text { def }}{=} R^{\varepsilon}(\hat{\theta}, \theta),
$$

where $\mathbf{E}_{\theta}$ denotes the expectation w.r.t. the distribution of the data in the model (3).

In this paper we consider the problem of estimation of $\theta$ in the model (3) using the mean-squared risk (4).

One can characterize linear inverse problems by the difficulty of the operator, i.e., with our notations, by the behavior of $b_{k}$ 's. If $b_{k} \rightarrow 0$, as $k \rightarrow \infty$, the problem is ill-posed. An inverse problem will be called softly ill-posed if the sequence $b_{k}$ tends to 0 at a polynomial rate in $k$ and it will be called severely ill-posed if

$$
\lim _{k \rightarrow \infty} \frac{1}{k} \log \frac{1}{b_{k}}=c
$$

for some $0<c<\infty$. Thus, the problem is severely ill-posed if, in the main term, $b_{k}$ tends to 0 exponentially in $k$.

An important element of the model is the prior information about $\theta$. Successful estimation of a sequence $\theta$ is possible only if its elements $\theta_{k}$ tend to zero sufficiently fast, as $k \rightarrow \infty$, which means that $f$ is sufficiently smooth. 
A standard assumption on the smoothness of $f$ is to suppose that $\theta$ belongs to an ellipsoid

$$
\Theta=\left\{\theta: \sum_{k=1}^{\infty} a_{k}^{2} \theta_{k}^{2} \leqslant L\right\},
$$

where $a=\left\{a_{k}\right\}$ is a positive sequence that tends to infinity, and $L>0$. Special cases of $\Theta$ are the Sobolev balls and the classes of analytic functions, corresponding to $a_{k}$ 's increasing as a polynomial in $k$ and as an exponential in $k$, respectively.

Thus appears a natural classification of different cases in the study of linear inverse problems. Regarding the difficulty of the operator described in terms of $b_{k}$ 's and the smoothness assumptions described in terms of $a_{k}$ 's, one obtains the following three typical cases.

$1^{\circ}$. Softly ill-posed problems: $b_{k}$ 's are polynomial and $a_{k}$ 's are general (usually polynomial or exponential). These problems have been studied by many authors, and they are essentially similar to estimation of derivatives of smooth functions. Sharp adaptive estimators for a general framework are given by Cavalier and Tsybakov [3] and by Cavalier, Golubev, Picard, and Tsybakov [2].

$2^{\circ}$. Severely ill-posed problems with log-rates: $b_{k}$ 's are exponential and $a_{k}$ 's are polynomial. This case is highly degenerate in the sense that the variance of the optimal estimators is asymptotically negligible as compared to their bias. The optimal rates of convergence are very slow (logarithmic) and sharp adaptation can be attained on a simple projection estimator $([4],[8])$.

$3^{\circ}$. 2 exp-severely ill-posed problems: $b_{k}$ 's are exponential and $a_{k}$ 's are exponential too (the abbreviation «2 exp» stands for «two exponentials»). These problems will be studied here. They are characterized by some unusual phenomena. Golubev and Khasminskii [9] proved that 2 exp problems admit fast optimal rates converging to 0 as a power law, despite the «severe» form of the operator. They also showed that sharp minimax estimators for these problems are nonlinear, unlike all other known cases where sharp minimaxity is explored. Also the adaptation issue turns out to be nonstandard here. As shown by Tsybakov [19], there is a logarithmic deterioration in the best rate of convergence for adaptive estimation under the $L_{2}$-risk. In other words, here one has to pay a price for $L_{2}$-adaptation, while this is not the case for the inverse problems described in $1^{\circ}$ and $2^{\circ}$ : there the $L_{2}$-adaptation is possible without any loss, and even the exact constants are preserved.

Since the ellipsoid $\Theta$ with exponential $a_{k}$ 's corresponds to analytic functions, the $2 \exp$ framework can be viewed as an analogue of convolution through a super-smooth filter (described by exponential $b_{k}$ 's), with an analytical function $f$ to reconstruct.

There is an important reason why the 2 exp setup is of interest. In the 
study of inverse problems, a standard assumption is to connect the smoothness of the underlying function to the smoothness of the operator. Roughly, if a function is observed through a very smooth filter, then the function itself has to be very smooth. A formalization of this idea can be found, for example, in the well-known Hilbert scale approach to inverse problems (see [7], [13]-[15]).

Nonadaptive minimax estimation for some inverse problems different from (3) but characterized by a similar «two exponentials» behavior has been analyzed by Ermakov [6], Pensky and Vidakovic [16], Efromovich and Koltchinskii [5].

In this paper we study estimation for the 2 exp framework when the ellipsoid $\Theta$ is not known, but we know only that $a_{k}$ 's are exponential. We propose an adaptive estimator which attains optimal rates (up to an inevitable logarithmic factor deterioration) simultaneously on all the ellipsoids with exponential $a_{k}$ 's. Moreover, we show that the estimator is sharp adaptive, i.e., it cannot be improved to within a constant. This generalizes the result of Tsybakov [19] about the optimal rate of adaptation for 2 exp problems. The construction of our adaptive estimator is based on a block thresholding (cf. [10] or [3] for the inverse problems setting). A difference from those papers is that, in order to get sharp optimality in our case, we need a «running» block estimator rather than an estimator with fixed blocks.

Let us give some examples of severely ill-posed inverse problems related to partial differential equations.

$\mathrm{E} \times \mathrm{a} \mathrm{m} \mathrm{pl} \mathrm{e} \mathrm{1.} \mathrm{Consider} \mathrm{the} \mathrm{Dirichlet} \mathrm{problem} \mathrm{for} \mathrm{the} \mathrm{Laplace} \mathrm{equa-}$ tion on a circle of radius 1 :

$$
\Delta u=0, \quad u(1, \varphi)=f(\varphi), \quad \varphi \in[0,2 \pi], \quad 0 \leqslant r \leqslant 1,
$$

where $\Delta$ is the Laplace operator, $u(r, \varphi)$ is a function in polar coordinates $r \geqslant 0, \varphi \in[0,2 \pi]$, and $f$ is a $2 \pi$-periodic function in $L_{2}[0,2 \pi]$. It is well known that the solution of (5) is

$$
u_{f}(r, \varphi)=\frac{\theta_{0}}{\sqrt{2 \pi}}+\frac{1}{\sqrt{\pi}} \sum_{k=1}^{\infty} r^{k}\left[\theta_{k} \cos (k \varphi)+\theta_{-k} \sin (k \varphi)\right]
$$

where $\theta_{k}$ are the Fourier coefficients of $f$. Assume that $f$ is not known, but one can observe the solution $u_{f}(r, \varphi)$ on the circle of radius $r_{0}<1$ in a white Gaussian noise:

$$
d Y(\varphi)=u_{f}\left(r_{0}, \varphi\right) d \varphi+\varepsilon d W(\varphi), \quad \varphi \in[0,2 \pi]
$$

where $W$ is a standard Wiener process on $[0,2 \pi]$ and $0<\varepsilon<1$. The problem is to estimate the boundary condition $f$ based on the observation of a trajectory $\{Y(\varphi), \varphi \in[0,2 \pi]\}$. Substituting (6) in (7), multiplying (7) by the trigonometric basis functions and integrating over $[0,2 \pi]$ we get the infinite sequence of observations $y_{k}=b_{k} \theta_{k}+\varepsilon \xi_{k}, k \in \mathbf{Z}$, where $b_{k}=r_{0}^{-|k|}$ and $\xi_{k}$ are i.i.d. $\mathscr{N}(0,1)$ random variables. By renumbering the indices from 
$k \in \mathbf{Z}$ to $k \in \mathbf{N}$ we get a particular case of the model (3). This problem is severely ill-posed since $b_{k} \rightarrow 0$ exponentially fast as $k \rightarrow \infty$.

Example 2. Consider the following Cauchy problem for the Laplace equation:

$$
\Delta u=0, \quad u(x, 0)=0,\left.\quad \frac{\partial}{\partial y} u(x, y)\right|_{y=0}=g(x),
$$

where $u(x, y)$ is defined for $x \in \mathbf{R}, y \geqslant 0$, and the initial condition $g$ is a 1-periodic function on $\mathbf{R}$.

Suppose that we do not know $g$ but we have in our disposal the noisy observations $\{Y(x), x \in[0,1]\}$, where $Y$ is the random process defined by

$$
d Y(x)=g(x) d x+\varepsilon d W(x), \quad x \in[0,1] .
$$

Here $W$ is the standard Wiener process on $[0,1]$. The problem is to estimate the solution $f(x) \stackrel{\text { def }}{=} u_{g}\left(x, y_{0}\right)$ of $(8)$ at a given $y_{0}>0$, based on these observations. Since $g$ is 1 -periodic, $f$ is also 1-periodic. Denoting $\theta_{k}$ the Fourier coefficients of $f$, one can find that, given (9), the following sequence of observations is available: $y_{k}=b_{k} \theta_{k}+\varepsilon \xi_{k}$, where $\xi_{k}$ are i.i.d. $\mathscr{N}(0,1)$ random variables and $b_{k} \sim k \exp \left(-\beta y_{0} k\right)$ as $k \rightarrow \infty$, for some $\beta>0$ (see [8] for more details).

2. Setting of the problem. From now on we assume that the observations have the form (3), where $\xi_{k}$ are i.i.d. $\mathscr{N}(0,1)$ random variables and the values $b_{k}$ are defined by

$$
b_{k}^{-2}=r_{k} \exp (\rho k)
$$

with $\rho>0$ and a positive sequence $r_{k}$ varying slower than an exponential as $k \rightarrow \infty$. Such a definition of $b_{k}$ covers the examples considered above, whereas considering the squared values of $b_{k}$ 's reflects the fact that the results will be insensitive to the signs.

We assume that $r_{k}$ is subexponential in the sense of the following definition.

Definition 1. A sequence $\left\{r_{k}\right\}_{k=1}^{\infty}$ is called subexponential if $r_{k}>0$ for all $k$ and there exist constants $C_{*}<\infty$ and $\mu \in(0,1]$ such that

$$
\left|\frac{r_{k+1}}{r_{k}}-1\right| \leqslant \frac{C_{*}}{k^{\mu}}, \quad k=1,2, \ldots
$$

The class of subexponential sequences is rather large, including polynomial, logarithmic and other sequences. It is easy to see that a subexponential sequence $r_{k}$ satisfies

$$
\begin{aligned}
& a \exp \left(-c k^{1-\mu}\right) \leqslant r_{k} \leqslant a^{\prime} \exp \left(c^{\prime} k^{1-\mu}\right) \quad \text { if } \quad 0<\mu<1, \\
& a k^{-c} \leqslant r_{k} \leqslant a^{\prime} k^{c^{\prime}} \quad \text { if } \mu=1,
\end{aligned}
$$

with some positive finite constants $a, a^{\prime}, c$, and $c^{\prime}$. 
We will assume that $\theta$ belongs to an ellipsoid

$$
\Theta(\alpha, L)=\left\{\theta: \sum_{k=1}^{\infty} q_{k} \exp (\alpha k) \theta_{k}^{2} \leqslant L\right\}
$$

where $q_{k}$ is a subexponential sequence and $\alpha>0, L>0$ are finite constants. In order to shed some light on the estimation of $\theta$ in this setup, consider a simple projection estimator $\widetilde{\theta}=\left(\widetilde{\theta}_{1}, \widetilde{\theta}_{2}, \ldots\right)$ with bandwidth $W \in \mathbf{N}$, i.e.,

$$
\tilde{\theta}_{k}= \begin{cases}b_{k}^{-1} y_{k}, & k \leqslant W \\ 0, & k>W .\end{cases}
$$

The maximal risk of this estimator over $\Theta(\alpha, L)$ is bounded from above as follows

$$
\begin{aligned}
\sup _{\theta \in \Theta(\alpha, L)} R^{\varepsilon}(\tilde{\theta}, \theta) & =\sup _{\theta \in \Theta(\alpha, L)} \sum_{k>W} \theta_{k}^{2}+\varepsilon^{2} \sum_{k=1}^{W} b_{k}^{-2} \\
& \leqslant L \sum_{k>W} \exp (-\alpha k) q_{k}^{-1}+\varepsilon^{2} \sum_{k=1}^{W} \exp (\rho k) r_{k} .
\end{aligned}
$$

The minimum of the right-hand side of (14) with respect to $W$ is attained for some $W$ depending on $\varepsilon$ such that $W \rightarrow \infty$ as $\varepsilon \rightarrow 0$ (in fact, otherwise the right-hand side of (14) does not tend to 0 as $\varepsilon \rightarrow 0$ ). Using Lemma 2 (see below) to compute the last two sums in (14), we find that as $W \rightarrow \infty$, the right hand-side of (14) is approximated by

$$
J(W)=\frac{L \exp (-\alpha W) q_{W}^{-1}}{1-\mathrm{e}^{-\alpha}}+\varepsilon^{2} \frac{\exp (\rho W) r_{W}}{1-\mathrm{e}^{-\rho}} .
$$

The minimizer of $J(W)$ gives an approximately optimal bandwidth. Since for subexponential sequences $r_{k}, q_{k}$ and large enough $W$ we have $r_{W-1} \approx r_{W} \approx$ $r_{W+1}, q_{W-1} \approx q_{W} \approx q_{W+1}$, the necessary conditions of a local minimum at $W$, namely $J(W)<J(W+1)$ and $J(W)<J(W-1)$, can be written in the form $L \exp (-\gamma W)<\varepsilon^{2} \mathrm{e}^{\rho} r_{W} q_{W}, L \exp (-\gamma(W-1))>\varepsilon^{2} \mathrm{e}^{\rho} r_{W-1} q_{W-1}$, where $\gamma=\rho+\alpha$. It can be shown that all the local minimizers of $J(W)$ provide essentially the same value of $J$, so that one can take, for example, the smallest local minimizer

$$
W(\alpha, L)=\min \left\{k \in \mathbf{N}: L \exp (-\gamma k)<\varepsilon^{2} \mathrm{e}^{\rho} r_{k} q_{k}\right\} .
$$

In other words, the minimum of the right-hand side of (14) is approximately attained at $W(\alpha, L)$. This yields the following upper bound for the minimax risk:

$$
\inf _{\hat{\theta}} \sup _{\theta \in \Theta(\alpha, L)} R^{\varepsilon}(\hat{\theta}, \theta) \leqslant C r_{\varepsilon}(\alpha, L),
$$

with a constant $C<\infty$ and the rate of convergence

$$
s_{\varepsilon}(\alpha, L)=\varepsilon^{2} r_{W(\alpha, L)} \exp [\rho W(\alpha, L)]
$$


Using the argument of [9] (see also Lemma 6 below) it is not difficult to show that the rate of convergence $s_{\varepsilon}(\alpha, L)$ cannot be improved in a minimax sense. Unfortunately, the minimax approach has a disadvantage: the minimax optimal (or nearly optimal) bandwidth depends on parameters $\alpha$ and $L$ of the functional class $\Theta(\alpha, L)$. Tsybakov [19] shows that one can overcome this difficulty and construct an adaptive estimator of $\theta$ independent of $\alpha$ and $L$ and attaining the rate which is only logarithmically worse than $s_{\varepsilon}(\alpha, L)$ on any class $\Theta(\alpha, L)$. In the next section we propose another adaptive estimator which attains the same rate as that of [19] but has an optimal asymptotic constant.

3. Adaptive estimator and its optimality. Define the following estimator $\theta^{*}=\left(\theta_{1}^{*}, \theta_{2}^{*}, \ldots\right)$ based on block thresholding with running blocks

$$
\theta_{k}^{*}=b_{k}^{-1} y_{k} \mathbf{I}\left\{\|y\|_{k}^{2} \geqslant 2 \varepsilon^{2} \rho^{\prime} k\right\}, \quad k=1,2, \ldots,
$$

where $\rho^{\prime}>\rho$ and

$$
\|y\|_{k}^{2}=\sum_{s \in \mathrm{N}:|k-s| \leqslant N} y_{s}^{2}
$$

with an integer $N \geqslant 1$. Here and later $\mathbf{I}\{\cdot\}$ denotes the indicator function.

It will be clear from the proofs that $\theta_{k}^{*}=0$ with a probability close to 1 , whenever $k$ does not belong to a «small» neighborhood of the integer $W^{*}$ defined by

$$
W^{*}=W^{*}(\alpha, L)=\min \left\{k \in \mathbf{N}: L \exp (-\gamma k)<2 \varepsilon^{2} \rho k r_{k} q_{k}\right\} .
$$

We will call $W^{*}$ the adaptive bandwidth. Note that $W^{*}(\alpha, L)$ is smaller than the optimal bandwidth $W(\alpha, L)$ given in (15) for all $\varepsilon$ small enough. For instance if $r_{k}=q_{k} \equiv 1$ we have as $\varepsilon \rightarrow 0$,

$$
W(\alpha, L)=\frac{1}{\gamma} \log \frac{L}{\varepsilon^{2}}+O(1)
$$

whereas

$$
W^{*}(\alpha, L)=\frac{1}{\gamma} \log \frac{L}{\varepsilon^{2}}-\frac{1}{\gamma} \log \log \frac{L}{\varepsilon^{2}}+O(1) .
$$

For general $r_{k}, q_{k}$ the closed form expression for $W^{*}$ is not available, but using (12) one can see that $W^{*} \asymp \log (1 / \varepsilon)$ as $\varepsilon \rightarrow 0$. Nevertheless, possible terms of the order $o(\log (1 / \varepsilon))$ in the expression for $W^{*}$ are not negligible since they can affect the rate of convergence (cf. (21)). Note also that the value $W^{*}$ need not be known for the construction of our estimator.

In this section we establish the exact asymptotics of the minimax adaptive risk. It turns out that this asymptotics is expressed in terms of the value $A^{*}$ of the following maximization problem:

$$
A^{*}=A^{*}(\alpha, L)=\max _{\theta \in \Theta_{\infty}(\alpha, L)} \sum_{k=-\infty}^{\infty} \exp (\rho k) \theta_{k}^{2},
$$


where

$$
\Theta_{\infty}(\alpha, L)=\left\{\theta \in \ell^{2}(\mathbf{Z}): \sum_{k=-\infty}^{\infty} \theta_{k}^{2} \leqslant 1, \sum_{k=-\infty}^{\infty} \exp (\gamma k) \theta_{k}^{2} \leqslant E^{*}(\alpha, L)\right\}
$$

and

$$
E^{*}(\alpha, L)=\frac{L \exp \left[-\gamma W^{*}(\alpha, L)\right]}{2 \varepsilon^{2} \rho W^{*}(\alpha, L) r_{W^{*}(\alpha, L)} q_{W^{*}(\alpha, L)}}
$$

Note that (22)-(23) is a problem of linear programming w.r.t. $\theta_{k}^{2}$ 's and it has a solution belonging to the boundary of $\Theta_{\infty}(\alpha, L)$. The values $A^{*}(\alpha, L)$ and $E^{*}(\alpha, L)$ depend on $\varepsilon$, but the dependence is not strong: they oscillate between two fixed constants as $\varepsilon$ varies. In fact, the definition of $W^{*}$ implies that for any $L$ and $\alpha$ there exist finite positive constants $e_{1}^{*}, e_{2}^{*}$ such that $e_{1}^{*} \leqslant E^{*}(\alpha, L) \leqslant e_{2}^{*}$ for all $\varepsilon$. This implies the existence of finite positive constants $a_{1}^{*}, a_{2}^{*}$ (depending on $L$ and $\alpha$ ) such that $a_{1}^{*} \leqslant A^{*}(\alpha, L) \leqslant a_{2}^{*}$ for all $\varepsilon$. In particular, since $\gamma>\rho$, one can take $a_{2}^{*}=e_{2}^{*}$.

Define

$$
\psi_{\varepsilon}(\alpha, L)=2 A^{*}(\alpha, L) \varepsilon^{2} \rho W^{* *}(\alpha, L) \exp \left[\rho W^{*}(\alpha, L)\right] r_{W^{*}(\alpha, L)} .
$$

The next theorem gives a bound for the maximal risk of the estimator $\theta^{*}$ over $\Theta(\alpha, L)$.

Theorem 1. Assume that $b_{k}$ satisfies (10) and that $r_{k}$ and $q_{k}$ are subexponential: Let $\theta^{*}$ be the estimator defined by (17)-(18) with $\rho^{\prime}>\rho$ and $N \in \mathbf{N}$. Then for any $\alpha>0$ and $L>0$ we have

$$
\limsup \sup _{\varepsilon \rightarrow 0} \frac{R^{\varepsilon}\left(\theta^{*}, \theta\right)}{\psi_{\varepsilon}(\alpha, L)} \leqslant \frac{\rho^{\prime}}{\rho}+C \exp \left[-\frac{N}{2} \min (\alpha, \rho)\right],
$$

where the constant $C<\infty$ does not depend on $N$ and $\rho^{\prime}$.

Note that if the size $2 N+1$ of the block is large and the parameter $\rho^{\prime}$ is close to $\rho$, then the right-hand side of (26) approaches 1 . Alternatively, one can take $N=N_{\varepsilon} \rightarrow \infty$ and $\rho^{\prime}=\rho_{\varepsilon}^{\prime} \rightarrow \rho$ as $\varepsilon \rightarrow 0$, satisfying appropriate restrictions, which leads to the next result. For $x \geqslant 0$ write $\lceil x\rceil=\min \{n \in \mathbf{N}$ : $n>x\}$.

Theorem 2. Assume that $b_{k}$ satisfies (10) and that $r_{k}$ and $q_{k}$ are subexponential. Let $\theta^{*}$ be the estimator defined by (17)-(18) with $N=$ $\lceil\sqrt{\log [\log (1 / \varepsilon) \vee 1]}]$ and $\rho^{\prime}=\rho+N^{-1}$. Then for any $\alpha>0$ and $L>0$ we have

$$
\limsup \sup _{\varepsilon \rightarrow 0} \frac{R^{\varepsilon}\left(\theta^{*}, \theta\right)}{\psi_{\varepsilon}(\alpha, L)} \leqslant 1 \text {. }
$$

$\mathrm{R}$ e $\mathrm{m}$ a $\mathrm{rk}$ 1. The estimator $\theta^{*}$ is defined as an infinite sequence. It can be proved that, under our assumptions, the number of non-zero components of this sequence is finite almost surely. However, formally the estimator (17) is not feasible since one has to check the inequality $\|y\|_{k}^{2} \geqslant 2 \varepsilon^{2} \rho^{\prime} k$ for 
all $k=1,2, \ldots$. This problem does not arise in practice where one always has a finite number $N_{\max }$ of coefficients to be dealt with (coming from intrinsic limitations of the data) and the estimator $\theta^{*}$ is modified by setting $\theta_{k}^{*}=0$ for all $k>N_{\max }$. Inspection of the proofs shows that to keep Theorems 1 and 2 valid for such a modified estimator it suffices to have $N_{\max } \geqslant \log ^{2}(1 / \varepsilon)$.

$\mathrm{R} \mathrm{e} \mathrm{m} \mathrm{a} \mathrm{r} \mathrm{k} \mathrm{2.} \mathrm{The} \mathrm{choice} \mathrm{of} N$ and $\rho$ suggested in Theorem 2 is not the only possible one: there exists a variety of similar values $(N, \rho)$ that allow to attain the result of the theorem. These values are described by technical conditions that we do not include in the theorem but that can easily be deduced from the proof.

The following theorem explains in what sense the estimator $\theta^{*}$ is optimal.

Theorem 3. Assume that $b_{k}$ satisfies (10) and that $r_{k}$ and $q_{k}$ are subexponential. Let an estimator $\hat{\theta}$ be such that, for some $\alpha_{0}>0, L_{0}>0$,

$$
\limsup _{\varepsilon \rightarrow 0} \sup _{\theta \in \Theta\left(\alpha_{0}, L_{0}\right)} \frac{R^{\varepsilon}(\hat{\theta}, \theta)}{\psi_{\varepsilon}\left(\alpha_{0}, L_{0}\right)}<1 .
$$

Then there exists $\alpha^{\prime}>\alpha_{0}$ such that for all $\alpha>\alpha^{\prime}$ and all $L>0$

$$
\liminf _{\varepsilon \rightarrow 0} \sup _{\theta \in \Theta\left(\alpha_{0}, L_{0}\right)} \frac{R^{\varepsilon}(\hat{\theta}, \theta)}{\psi_{\varepsilon}\left(\alpha_{0}, L_{0}\right)} \sup _{\theta \in \Theta(\alpha, L)} \frac{R^{\varepsilon}(\hat{\theta}, \theta)}{\psi_{\varepsilon}(\alpha, L)}=\infty .
$$

Theorems 2 and 3 imply in particular that $\psi_{\varepsilon}(\alpha, L)$ is an adaptive rate of convergence for our problem (cf. Definition 3 in [18]). But they give more than just the rate. These theorems show that if an estimator $\hat{\theta}$ gains over $\theta^{*}$ at one point $\left(\alpha_{0}, L_{0}\right)$ at least in terms of a constant factor (cf. (28)), there exists another point $(\alpha, L)$ where $\hat{\theta}$ looses much more than it gains at $\left(\alpha_{0}, L_{0}\right)$ (cf. (29)). One can interpret this property as sharp adaptive optimality of the normalizing factor $\psi_{\varepsilon}(\cdot, \cdot)$ and, consequently, of the estimator $\theta^{*}$ whose convergence is characterized by this normalizing factor.

Note also that for every fixed $\alpha_{0}>0, L_{0}>0$ there exists an estimator $\hat{\theta}$ satisfying (28) and even more:

$$
\limsup _{\varepsilon \rightarrow 0} \sup _{\theta \in \Theta\left(\alpha_{0}, L_{0}\right)} \frac{R^{\varepsilon}(\hat{\theta}, \theta)}{\psi_{\varepsilon}\left(\alpha_{0}, L_{0}\right)}=0 .
$$

For example, one can take $\hat{\theta}$ as a projection estimator with the optimal bandwidth corresponding to $\left(\alpha_{0}, L_{0}\right)$ (see Section 2). Thus, $\hat{\theta}$ gains over $\theta^{*}$ «at a point» $\left(\alpha_{0}, L_{0}\right)$. But at any other point $(\alpha, L)$ with $\alpha>\alpha_{0}$ this estimator has catastrophic behavior (cf. (29)).

For a more illustrative interpretation, one can modify Theorem 3 by expressing the result in terms of the ratio of maximal risks. For any two estimators $\hat{\theta}_{1}$ and $\hat{\theta}_{2}$ define

$$
G_{\alpha, L}\left(\hat{\theta}_{1} / \hat{\theta}_{2}\right)=\frac{\sup _{\theta \in \Theta(\alpha, L)} R^{\varepsilon}\left(\hat{\theta}_{2}, \theta\right)}{\sup _{\theta \in \Theta(\alpha, L)} R^{\varepsilon}\left(\hat{\theta}_{1}, \theta\right)} .
$$


This value is interpreted as the gain of $\hat{\theta}_{1}$ over $\hat{\theta}_{2}$ at $(\alpha, L)$. The larger is $G_{\alpha, L}\left(\hat{\theta}_{1} / \hat{\theta}_{2}\right)$, the better is $\hat{\theta}_{1}$ as compared to $\hat{\theta}_{2}$. It is easy to see that Theorem 3 and (27) imply the following corollary.

Corollary 1. Under assumptions of Theorem 3, let an estimator $\hat{\theta}$ be such that, for some $\alpha_{0}>0, L_{0}>0$,

$$
\liminf _{\varepsilon \rightarrow 0} G_{\alpha_{0}, L_{0}}\left(\hat{\theta} / \theta^{*}\right)>1 \text {, }
$$

where $\theta^{*}$ is the estimator defined by (17)-(18) and satisfying the assumptions of Theorem 2. Then there exists $\alpha>\alpha_{0}$ such that for all $L>0$ and all $\varepsilon$ small enough

$$
G_{\alpha, L}\left(\theta^{*} / \hat{\theta}\right)>\ell_{\varepsilon} G_{\alpha_{0}, L_{0}}\left(\hat{\theta} / \theta^{*}\right)
$$

with $\ell_{\varepsilon} \rightarrow \infty$, as $\varepsilon \rightarrow 0$.

$\mathrm{R}$ e $\mathrm{m}$ a r k 3. Consider, for instance, the case where $r_{k}$ and $q_{k}$ do not depend on $k$. Then it follows from (15) and (16) that the nonadaptive rate of convergence $s_{\varepsilon}(\alpha, L)$ is of the order $\varepsilon^{2 \alpha / \gamma}$, while (21) and (25) imply that the adaptive rate satisfies $\psi_{\varepsilon}(\alpha, L) \asymp \varepsilon^{2 \alpha / \gamma} \log (1 / \varepsilon)^{\alpha / \gamma}$. Thus, one has to pay an extra log-factor for adaptation. This effect is similar to the one established by Lepski [12] for adaptation using the loss at a fixed point, and it is due to a nondegenerate asymptotic behavior of the normalized loss of the estimators as $\varepsilon \rightarrow 0$. It is interesting to note that our problem provides an example where such an effect occurs for adaptation using the $L_{2}$-loss.

4. Proofs. In this section we will denote by $C$ finite positive constants that may be different in different occasions.

\subsection{Proof of Theorems 1 and 2.}

Lemma 1. Let $w_{k}$ be a subexponential sequence. Then for any integers $T, t$ such that $t \geqslant T$ and any integer $M<\min (T, \log T)$ we have

$$
\sup _{k \in \mathbf{Z}:|k| \leqslant M}\left|\frac{w_{t+k}}{w_{t}}-1\right| \leqslant \eta_{T}
$$

where $\eta_{T}$ depends only on $T$ and $\eta_{T} \rightarrow 0$ as $T \rightarrow \infty$.

$\mathrm{P}$ r o of is straightforward.

Lemma 2. Let $w_{k}$ be a subexponential sequence. Then for any $\tau>0$ as $T \rightarrow \infty$

$$
\begin{aligned}
\sum_{k=1}^{T} \exp (\tau k) w_{k} & =(1+o(1)) \frac{\exp (\tau T) w_{T}}{1-\mathrm{e}^{-\tau}} \\
\sum_{k=T}^{\infty} \exp (-\tau k) w_{k} & =(1+o(1)) \frac{\exp (-\tau T) w_{T}}{1-\mathrm{e}^{-\tau}} .
\end{aligned}
$$

P r o of. Write

$$
\sum_{k=1}^{T} \exp (\tau k) w_{k}=\exp (\tau T) w_{T} \sum_{k=0}^{T-1} \exp (-\tau k) \frac{w_{T-k}}{w_{T}} .
$$


Set $M=\lceil\log T\rceil-1$. If $T$ is large, we have $M<T-1$, and hence we can write

$$
\sum_{k=0}^{T-1} \exp (-\tau k) \frac{w_{T-k}}{w_{T}}=\sum_{k=0}^{M} \exp (-\tau k) \frac{w_{T-k}}{w_{T}}+\sum_{k=M+1}^{T-1} \exp (-\tau k) \frac{w_{T-k}}{w_{T}} .
$$

Using Lemma 1, we get

$$
\left(1-\eta_{T}\right) \sum_{k=0}^{M} \exp (-\tau k) \leqslant \sum_{k=0}^{M} \exp (-\tau k) \frac{w_{T-k}}{w_{T}} \leqslant\left(1+\eta_{T}\right) \sum_{k=0}^{M} \exp (-\tau k),
$$

where $\eta_{T}=o(1)$ as $T \rightarrow \infty$. Thus

$$
\lim _{T \rightarrow \infty} \sum_{k=0}^{M} \exp (-\tau k) \frac{w_{T-k}}{w_{T}}=\frac{1}{1-\mathrm{e}^{-\tau}} .
$$

The last sum in (34) satisfies

$$
\begin{aligned}
\sum_{k=M+1}^{T-1} \exp (-\tau k) \frac{w_{T-k}}{w_{T}} & \leqslant \sum_{k=M+1}^{T-1} \exp (-\tau k)\left(1+\frac{C}{M^{\mu}}\right)^{k} \\
& \leqslant \sum_{k=M+1}^{\infty} \exp \left\{-\left(\tau-C M^{-\mu}\right) k\right\} .
\end{aligned}
$$

This term tends to 0 as $M \rightarrow \infty$. Thus we obtain (32). Equation (33) is proved similarly.

Lemma 3. Let $\xi_{i}$ be i.i.d. $\mathscr{N}(0,1)$ random variables. Then, for any $k \in \mathbf{N}, N \in \mathbf{N}$ and $x>0$,

$$
\mathbf{E} \xi_{k}^{2} \mathbf{I}\left\{\|\xi\|_{k}^{2} \geqslant x\right\} \leqslant\left(\frac{x \mathrm{e}^{2}}{3+2 N}\right)^{N+3 / 2} \exp \left(-\frac{x}{2}\right) .
$$

P r o o f. For any $0<\lambda<\frac{1}{2}$,

$$
\begin{aligned}
\mathbf{E} \xi_{k}^{2} \mathbf{I}\left\{\|\xi\|_{k}^{2} \geqslant x\right\} & \leqslant \exp (-\lambda x) \mathbf{E} \xi_{k}^{2} \exp \left\{\lambda\|\xi\|_{k}^{2}\right\} \\
& =\exp (-\lambda x) \mathbf{E} \xi_{k}^{2} \exp \left\{\lambda \xi_{k}^{2}\right\} \prod_{i \neq k} \exp \left(\lambda \xi_{1}^{2}\right) \\
& =\exp (-\lambda x)(1-2 \lambda)^{-3 / 2}(1-2 \lambda)^{-N} \\
& =\exp \left(-\lambda x-\frac{3+2 N}{2} \log (1-2 \lambda)\right) .
\end{aligned}
$$

The minimum with respect to $\lambda$ of the right-hand side of (36) is attained at $\lambda=\frac{1}{2}(1-(3+2 N) / x)$. Substituting this $\lambda$ into (36) we get (35).

$\mathrm{P}$ r o of of $\mathrm{T}$ h e or e $\mathrm{m} 1$. Let $M$ be a sufficiently large integer satisfying $N \leqslant M<\min \left(W^{*} / 2, \log W^{*} / 2\right)$. In this proof we denote by $C$ the constants that do not depend on $M, N, \varepsilon$, and $\theta$. 
We decompose the risk of the estimator $\theta^{*}$ into three parts

$$
\sup _{\theta \in \Theta(\alpha, L)} R^{\varepsilon}\left(\theta^{*}, \theta\right) \leqslant S_{1}+S_{2}+S_{3},
$$

where

$$
\begin{aligned}
S_{1} & =\sup _{\theta \in \Theta(\alpha, L)} \sum_{k=1}^{W^{*}-M} \mathbf{E}_{\theta}\left(\theta_{k}^{*}-\theta_{k}\right)^{2}, \quad S_{2}=\sup _{\theta \in \Theta(\alpha, L)} \sum_{k=W^{*}-M}^{W^{*}+M} \mathbf{E}_{\theta}\left(\theta_{k}^{*}-\theta_{k}\right)^{2}, \\
S_{3} & =\sup _{\theta \in \Theta(\alpha, L)} \sum_{k=W^{*}+M}^{\infty} \mathbf{E}_{\theta}\left(\theta_{k}^{*}-\theta_{k}\right)^{2} .
\end{aligned}
$$

Consider first the term $S_{1}$. Using (32) for the subexponential sequences $w_{k}=r_{k}$ and $w_{k}=k r_{k}$, and Lemma 1 for $w_{k}=r_{k}$, we have

$$
\begin{aligned}
S_{1}= & \sup _{\theta \in \Theta(\alpha, L)} \sum_{k=1}^{W^{*}-M} \exp (\rho k) r_{k} \mathbf{E}_{\theta}\left[y_{k} \mathbf{I}\left\{\|y\|_{k}^{2}<2 \varepsilon^{2} \rho^{\prime} k\right\}-\varepsilon \xi_{k}\right]^{2} \\
\leqslant & 2 \varepsilon^{2} \sum_{k=1}^{W^{*}-M} \exp (\rho k) r_{k} \\
& +2 \sup _{\theta \in \Theta(\alpha, L)} \sum_{k=1}^{W^{*}-M} \exp (\rho k) r_{k} \mathbf{E}_{\theta} y_{k}^{2} \mathbf{I}\left\{\|y\|_{k}^{2}<2 \varepsilon^{2} \rho^{\prime} k\right\} \\
\leqslant & 2 \varepsilon^{2} \sum_{k=1}^{W^{*}-M} \exp (\rho k) r_{k}+4 \varepsilon^{2} \sum_{k=1}^{W^{*}-M} \exp (\rho k) r_{k} k \rho^{\prime} \\
\leqslant & C \rho \varepsilon^{2} \exp \left(\rho W^{*}\right) r_{W^{*}} W^{*} \exp (-\rho M) \leqslant C \psi_{\varepsilon}(\alpha, L) \exp (-\rho M) .
\end{aligned}
$$

Next, we bound from above the term $S_{2}$. This is the main term in (37) and we will analyze it using a renormalization argument. We begin with some simple remarks. Denote

$$
\Theta_{M}=\left\{\theta=\left(\theta_{1}, \theta_{2}, \ldots\right): \sum_{k=W^{*}-M-N}^{W^{*}+M+N} \exp (\gamma k) q_{k} \theta_{k}^{2} \leqslant L\right\} .
$$

Clearly, $\Theta(\alpha, L) \subset \Theta_{M}$. Now we change the variables from $\theta_{k}$ to $\nu_{k}$ by setting, for $k \geqslant 1-W^{*}$,

$\nu_{k}=\frac{\theta_{k+W^{*}}}{\varepsilon \sqrt{2 \rho W^{*} r_{k+W^{*}} \exp \left(\rho\left(k+W^{*}\right)\right)}}=\theta_{k+W^{*}}\left(\frac{A^{*} r_{W^{*}}}{\psi_{\varepsilon}(\alpha, L) \exp (\rho k) r_{k+W^{*}}}\right)^{1 / 2}$,

and let $\nu_{k}^{*}$ be derived from $\theta_{k}^{*}$ by the same transformation. If $\theta \in \Theta_{M}$, the sequence $\nu=\left\{\nu_{k}\right\}$ belongs to the set

$$
\Xi_{M}=\left\{\nu \in \Xi: \sum_{k=-M-N}^{M+N} \exp (\gamma k) r_{k+W^{*}} q_{k+W^{*}} \nu_{k}^{2} \leqslant \frac{L \exp \left(-\gamma W^{*}\right)}{2 \varepsilon^{2} \rho W^{*}}\right\}
$$

where $\Xi$ is the set of all sequences of the form $\nu=\left(\nu_{1-W^{*}}, \ldots, \nu_{0}, \nu_{1}, \ldots\right)$. Now, in view of Lemma 1, applied to the subexponential sequences $w_{k}=r_{k}$ 
and $w_{k}=r_{k} q_{k}$, there exists $\eta=\eta_{\varepsilon}$ depending only on $W^{*}$, such that $\eta \rightarrow 0$, as $\varepsilon \rightarrow 0$, and

$$
\begin{gathered}
\max _{|k| \leqslant M} r_{k+W^{*}} \leqslant(1+\eta) r_{W^{*}}, \\
\min _{|k| \leqslant M+N} r_{k+W^{*}} q_{k+W^{*}} \geqslant(1-\eta) r_{W^{*}} q_{W^{*}}
\end{gathered}
$$

Fix $0<\delta<1$, and assume that $\varepsilon$ is small enough to have simultaneously $\eta<\delta$ and $(1-\eta)^{-1}<1+\delta$. Then relation (40) implies that $\min _{|k| \leqslant M+N} r_{k+W^{*}} q_{k+W^{*}} \geqslant r_{W^{*}} q_{W^{*}} /(1+\delta)$, and therefore

$$
\Xi_{M} \subset \Xi_{M}^{\delta}=\left\{\nu \in \Xi: \sum_{k=-M-N}^{M+N} \exp (\gamma k) \nu_{k}^{2} \leqslant E^{*}(1+\delta)\right\} .
$$

Furthermore, (39) guarantees that $\max _{|k| \leqslant M} r_{k+W^{*}} \leqslant(1+\delta) r_{W^{*}}$. These remarks imply that, for $\varepsilon$ small enough,

$$
\begin{aligned}
S_{2} & \leqslant \sup _{\theta \in \Theta_{M}} \sum_{k=W^{*}-M}^{W^{*}+M} \mathbf{E}_{\theta}\left(\theta_{k}^{*}-\theta_{k}\right)^{2} \\
& \leqslant \frac{\psi_{\varepsilon}(\alpha, L)}{A^{*}} \sup _{\nu \in \Xi_{M}^{\delta}} \sum_{k=-M}^{M} \exp (\rho k) \frac{r_{k+W^{*}}}{r_{W^{*}}} \mathbf{E}_{\theta}\left(\nu_{k}^{*}-\nu_{k}\right)^{2} \\
& \leqslant \frac{(1+\delta) \psi_{\varepsilon}(\alpha, L)}{A^{*}} \sup _{\nu \in \Xi_{M}^{\delta}} \sum_{k=-M}^{M} \exp (\rho k) \mathbf{E}_{\theta}\left(\nu_{k}^{*}-\nu_{k}\right)^{2} .
\end{aligned}
$$

Using the inequality $(x+y)^{2} \leqslant(1+\delta) x^{2}+\left(1+\delta^{-1}\right) y^{2}$ for any $x, y \in \mathbf{R}$, we get

$$
\begin{aligned}
\mathbf{E}_{\theta}\left(\nu_{k}^{*}-\nu_{k}\right)^{2}= & \mathbf{E}\left[\left(\nu_{k}+\frac{\xi_{k+W^{*}}}{\sqrt{2 \rho W^{*}}}\right)\right. \\
& \left.\quad \times \mathbf{I}\left\{\sum_{l=k-N}^{k+N}\left(\nu_{l}+\frac{\xi_{l+W^{*}}}{\sqrt{2 \rho W^{*}}}\right)^{2} \geqslant \frac{\rho^{\prime}}{\rho}\left(1+\frac{k}{W^{*}}\right)\right\}-\nu_{k}\right]^{2} \\
\leqslant & (1+\delta) \nu_{k}^{2} \mathbf{P}\left\{\sum_{l=k-N}^{k+N}\left(\nu_{l}+\frac{\xi_{l+W^{*}}}{\sqrt{2 \rho W^{*}}}\right)^{2}<\frac{\rho^{\prime}}{\rho}\left(1+\frac{k}{W^{*}}\right)\right\} \\
& +\left(1+\delta^{-1}\right)\left(2 \rho W^{*}\right)^{-1} .
\end{aligned}
$$

Next, using the inequality

$$
(x+y)^{2} \geqslant(1-\delta) x^{2}+\left(1-\delta^{-1}\right) y^{2} \quad \text { for any } \quad x, y \in \mathbf{R},
$$

one obtains

$$
\sum_{l=k-N}^{k+N}\left(\nu_{l}+\frac{\xi_{l+W^{*}}}{\sqrt{2 \rho W^{*}}}\right)^{2} \geqslant(1-\delta) \sum_{l=k-N}^{k+N} \nu_{l}^{2}-\frac{1-\delta}{2 \rho W^{*} \delta} \sum_{l=k-N}^{k+N} \xi_{l+W^{*}}^{2}
$$


and, therefore,

$$
\begin{aligned}
& \mathbf{P}\left\{\sum_{l=k-N}^{k+N}\left(\nu_{l}+\frac{\xi_{l+W^{*}}}{\sqrt{2 \rho W^{*}}}\right)^{2}<\frac{\rho^{\prime}}{\rho}\left(1+\frac{k}{W^{*}}\right)\right\} \\
& \quad \leqslant \mathbf{P}\left\{(1-\delta) \sum_{l=k-N}^{k+N} \nu_{l}^{2}-\frac{1-\delta}{2 \rho W^{*} \delta} \sum_{l=k-N}^{k+N} \xi_{l+W^{*}}^{2}<\frac{\rho^{\prime}}{\rho}\left(1+\frac{k}{W^{*}}\right)\right\} \\
& \quad \leqslant \mathbf{I}\left\{\sum_{l=k-N}^{k+N} \nu_{l}^{2} \leqslant V\right\}+\mathbf{P}\left\{\frac{1}{4 \delta \rho W^{*}} \sum_{l=k-N}^{k+N} \xi_{l+W^{*}}^{2}>\delta\right\},
\end{aligned}
$$

where

$$
V=2 \delta+\frac{1}{1-\delta}\left(1+\frac{M}{W^{*}}\right) \frac{\rho^{\prime}}{\rho} .
$$

By Markov's inequality

$$
\mathbf{P}\left\{\frac{1}{4 \delta \rho W^{*}} \sum_{l=k-N}^{k+N} \xi_{l+W^{*}}^{2}>\delta\right\} \leqslant \frac{2 N+1}{4 \delta^{2} \rho W^{*}} .
$$

Define

$$
A(N, M)=\sup _{\nu \in \Xi_{M}^{\delta}} \sum_{k=-M}^{M} \exp (\rho k) \nu_{k}^{2} \mathbf{I}\left\{\sum_{l=k-N}^{k+N} \nu_{l}^{2} \leqslant V\right\} .
$$

Combining (42)-(44) one obtains

$$
\begin{aligned}
& \sup _{\nu \in \Xi_{M}^{\delta}} \sum_{k=-M}^{M} \exp (\rho k) \mathbf{E}_{\theta}\left(\nu_{k}^{*}-\nu_{k}\right)^{2} \leqslant(1+\delta) \\
& +\left[A(N, M)+\frac{2 N+1}{4 \delta^{2} \rho W^{*}} \sup _{\nu \in \Xi_{M}^{\delta}} \sum_{k=-M}^{M} \exp (\rho k) \nu_{k}^{2}\right]+\frac{C \exp (\rho M)}{W^{*}} \\
& \quad \leqslant(1+\delta) A(N, M)+\frac{C}{W^{*}}(N+\exp (\rho M)),
\end{aligned}
$$

where for the last inequality we used that, since $\gamma>\rho$,

$$
\sup _{\nu \in \Xi_{M}^{\delta}} \sum_{k=-M}^{M} \exp (\rho k) \nu_{k}^{2} \leqslant \sup _{\nu \in \Xi_{M}^{\delta}} \sum_{k=-M}^{M} \exp (\gamma k) \nu_{k}^{2} \leqslant E^{*}(1+\delta) .
$$

From (41) and (45) we get that for any $0<\delta<1$ and for all $\varepsilon$ small enough

$$
S_{2} \leqslant \frac{\psi_{\varepsilon}(\alpha, L)}{A^{*}}\left[(1+\delta)^{2} A(N, M)+\frac{C(N+\exp (\rho M))}{W^{*}}\right] .
$$

Our next goal is to show that $A(N, M)$ is close to $A^{*}$. We will proceed in steps. The first step is to remark that

$$
\begin{aligned}
A(N, M) & \leqslant \sup _{\nu \in \Xi_{M}^{\delta}} \sum_{k=-M}^{M} \exp (\rho k) \nu_{k}^{2} \mathbf{I}\left\{\sum_{l=k-N}^{k+N} \nu_{l}^{2} \leqslant V(1+\delta)\right\} \\
& \leqslant V\left[\sup _{\nu \in \Xi_{*}} \sum_{k=-M}^{M} \exp (\rho k) \nu_{k}^{2} \mathbf{I}\left\{\sum_{l=k-N}^{k+N} \nu_{l}^{2} \leqslant 1\right\}\right],
\end{aligned}
$$


where

$$
\Xi_{*}=\left\{\nu \in \Xi: \sum_{k=-M-N}^{M+N} \exp (\gamma k) \nu_{k}^{2} \leqslant E^{*}\right\} .
$$

In fact, to get (47), introduce the sequence $\nu^{\prime} \in \Xi$ such that $\nu_{k}=$ $\nu_{k}^{\prime} \sqrt{V(1+\delta)}$, observe that $V>1$ and use the embedding

$$
\begin{aligned}
& \left\{\nu^{\prime} \in \Xi: V \sum_{k=-M-N}^{M+N} \exp (\gamma k)\left(\nu_{k}^{\prime}\right)^{2} \leqslant E^{*}\right\} \\
& \subseteq\left\{\nu^{\prime} \in \Xi: \sum_{k=-M-N}^{M+N} \exp (\gamma k)\left(\nu_{k}^{\prime}\right)^{2} \leqslant E^{*}\right\} .
\end{aligned}
$$

Our next step is to find an upper bound for the expression in square brackets in (47). We can write assuming without loss of generality that $N$ is even,

$$
\sup _{\nu \in \Xi_{*}} \sum_{k=-M-N}^{M+N} \exp (\rho k) \nu_{k}^{2} \mathbf{I}\left\{\sum_{l=k-N}^{k+N} \nu_{l}^{2} \leqslant 1\right\} \leqslant A_{1}+A_{2}+A_{3}
$$

with

$$
\begin{aligned}
A_{1} & =\sup _{\nu \in \Xi_{*}} \sum_{k=-M-N}^{-N / 2-1} \exp (\rho k) \nu_{k}^{2} \mathbf{I}\left\{\sum_{l=k-N}^{k+N} \nu_{l}^{2} \leqslant 1\right\} \leqslant \sum_{k=-\infty}^{-N / 2-1} \mathrm{e}^{\rho k} \\
& \leqslant C \exp \left(-\frac{\rho N}{2}\right), \\
A_{2} & =\sup _{\nu \in \Xi_{*}} \sum_{k=N / 2+1}^{M+N} \exp (\rho k) \nu_{k}^{2} \mathbf{I}\left\{\sum_{l=k-N}^{k+N} \nu_{l}^{2} \leqslant 1\right\} \leqslant \sup _{\nu \in \Xi_{*}} \sum_{k=N / 2+1}^{M+N} \mathrm{e}^{\rho k} \nu_{k}^{2} \\
& \leqslant C \exp \left(-\frac{\alpha N}{2}\right),
\end{aligned}
$$

and

$$
\begin{aligned}
A_{3} & =\sup _{\nu \in \Xi_{*}} \sum_{k=-N / 2}^{N / 2} \exp (\rho k) \nu_{k}^{2} \mathbf{I}\left\{\sum_{l=k-N}^{k+N} \nu_{l}^{2} \leqslant 1\right\} \\
& \leqslant \sup _{\nu \in \Xi_{*}} \sum_{k=-N / 2}^{N / 2} \exp (\rho k) \nu_{k}^{2} \mathbf{I}\left\{\sum_{l=-N / 2}^{N / 2} \nu_{l}^{2} \leqslant 1\right\} \leqslant \sup _{\nu \in \Xi^{\prime}} \sum_{k=-N / 2}^{N / 2} \exp (\rho k) \nu_{k}^{2},
\end{aligned}
$$

where $\Xi^{\prime}=\left\{\nu \in \Xi: \sum_{k=-M-N}^{M+N} \exp (\gamma k) \nu_{k}^{2} \leqslant E^{*}, \sum_{k=-N / 2}^{N / 2} \nu_{k}^{2} \leqslant 1\right\}$. Substitution of the inequalities for $A_{1}, A_{2}$, and $A_{3}$ into (48) yields

$$
\begin{aligned}
& \sup _{\nu \in \Xi_{*}} \sum_{k=-M-N}^{M+N} \exp (\rho k) \nu_{k}^{2} \mathbf{I}\left\{\sum_{l=k-N}^{k+N} \nu_{l}^{2} \leqslant 1\right\} \\
& \leqslant \sup _{\nu \in \Xi^{\prime}} \sum_{k=-N / 2}^{N / 2} \exp (\rho k) \nu_{k}^{2}+C \exp \left(-\frac{N}{2} \min (\alpha, \rho)\right) .
\end{aligned}
$$


Next, introducing the set

$$
\Theta_{\infty}^{N / 2}(\alpha, L)=\left\{\nu \in \Theta_{\infty}(\alpha, L): \nu_{k}=0, \text { for }|k|>\frac{N}{2}\right\}
$$

we note that

$$
\begin{aligned}
\sup _{\nu \in \Xi^{\prime}} \sum_{k=-N / 2}^{N / 2} \exp (\rho k) \nu_{k}^{2} & =\sup _{\nu \in \Theta_{\infty}^{N / 2}(\alpha, L)} \sum_{k=-N / 2}^{N / 2} \exp (\rho k) \nu_{k}^{2} \\
& \leqslant \sup _{\nu \in \Theta_{\infty}(\alpha, L)} \sum_{k=-N / 2}^{N / 2} \exp (\rho k) \nu_{k}^{2} \leqslant A^{*} .
\end{aligned}
$$

In fact, the equality in (50) follows from the fact that taking $\nu_{k} \neq 0$ for $|k|>N / 2$ does not increase the sum $\sum_{k=-N / 2}^{N / 2} \exp (\rho k) \nu_{k}^{2}$, and thus the supremum of this sum over $\Xi^{\prime}$ is attained on the sequences $\nu$ with $\nu_{k}=0$ for $|k|>N / 2$.

From (47), (49)-(50) we get

$$
A(N, M) \leqslant V\left[A^{*}+C \exp \left(-\frac{N}{2} \min (\alpha, \rho)\right)\right] .
$$

This together with (46) and fact that $A^{*}$ is bounded from below uniformly in $\varepsilon$ entail

$$
\begin{gathered}
S_{2} \leqslant \psi_{\varepsilon}(\alpha, L)\left[V(1+\delta)^{2}+C \exp \left(-\frac{N}{2} \min (\alpha, \rho)\right)\right. \\
\left.+\frac{C}{W^{*}}(N+\exp (\rho M))\right]
\end{gathered}
$$

Finally we bound from above the term $S_{3}$. Using (3) and (10) we find

$$
\begin{aligned}
S_{3} \leqslant & 2 \sup _{\theta \in \Theta(\alpha, L)} \sum_{k=W^{*}+M}^{\infty} \theta_{k}^{2} \\
& +2 \varepsilon^{2} \sup _{\theta \in \Theta(\alpha, L)} \sum_{k=W^{*}+M}^{\infty} \exp (\rho k) r_{k} \mathbf{E} \xi_{k}^{2} \mathbf{I}\left\{\|y\|_{k}^{2} \geqslant 2 \varepsilon^{2} \rho^{\prime} k\right\} .
\end{aligned}
$$

The first term in the right-hand side satisfies

$$
\begin{gathered}
\sup _{\theta \in \Theta(\alpha, L)} \sum_{k=W^{*}+M}^{\infty} \theta_{k}^{2}=\sup _{\theta \in \Theta(\alpha, L)} \sum_{k=W^{*}+M}^{\infty} \exp (-\alpha k) q_{k}^{-1} q_{k} \theta_{k}^{2} \exp (\alpha k) \\
\leqslant \sup _{\theta \in \Theta(\alpha, L)} \sum_{k=W^{*}+M}^{\infty} \exp (-\alpha k) q_{k}^{-1} \sum_{k=1}^{\infty} q_{k} \exp (\alpha k) \theta_{k}^{2} \\
\leqslant C \exp \left[-\alpha\left(W^{*}+M\right)\right] q_{W^{*}+M}^{-1} \leqslant C \exp [-\alpha M] \psi_{\varepsilon}(\alpha, L),
\end{gathered}
$$

where to get the last two inequalities we applied Lemma 2 and then Lemma 1 for the subexponential sequence $w_{k}=q_{k}^{-1}$. 
Consider the sequence $\theta^{\prime}=\left(\theta_{1}^{\prime}, \theta_{2}^{\prime}, \ldots\right)$ with $\theta_{k}^{\prime}=b_{k} \theta_{k}$. For $k \geqslant W^{*}+M$

$$
\begin{aligned}
\sup _{\theta \in \Theta(\alpha, L)}\left\|\theta^{\prime}\right\|_{k}^{2} & =\sup _{\theta \in \Theta(\alpha, L)} \sum_{l=k-N}^{k+N} \theta_{l}^{2} r_{l}^{-1} \exp (-\rho l) \\
& \leqslant \sup _{\theta \in \Theta(\alpha, L)} \sum_{l=k-N}^{k+N} q_{l} \exp (\alpha l) \theta_{l}^{2} \sum_{l=k-N}^{k+N} r_{l}^{-1} q_{l}^{-1} \exp (-\gamma l) \\
& \leqslant L \sum_{l=k-N}^{k+N} r_{l}^{-1} q_{l}^{-1} \exp (-\gamma l) \leqslant C r_{k-N}^{-1} q_{k-N}^{-1} \exp [-\gamma(k-N)],
\end{aligned}
$$

where we used Lemma 2 for the subexponential sequence $w_{l}=r_{l}^{-1} q_{l}^{-1}$. Applying to the last expression of the previous display successively Lemma 1 and then the fact that

$$
L \exp \left(-\gamma W^{*}\right)<2 \varepsilon^{2} \rho W^{*} r_{W^{*}} q_{W^{*}},
$$

we get

$$
\begin{aligned}
\sup _{\theta \in \Theta(\alpha, L)}\left\|\theta^{\prime}\right\|_{k}^{2} & \leqslant C r_{W^{*}}^{-1} q_{W^{*}}^{-1} \exp (-\gamma(k-N)) \\
& \leqslant C \varepsilon^{2} W^{*} \exp (-\gamma(M-N)) \leqslant C \varepsilon^{2} k \exp (-\gamma(M-N)) .
\end{aligned}
$$

Now we bound the last term in (52). By Lemma 3 , for any $k \geqslant W^{*}+M$ we have

$$
\begin{aligned}
& \mathbf{E} \xi_{k}^{2} \mathbf{I}\left\{\left\|\theta^{\prime}+\varepsilon \xi\right\|_{k}^{2} \geqslant 2 \varepsilon^{2} \rho^{\prime} k\right\} \leqslant \mathbf{E} \xi_{k}^{2} \mathbf{I}\left\{\|\xi\|_{k} \geqslant \sqrt{2 \rho^{\prime} k}-\left\|\frac{\theta^{\prime}}{\varepsilon}\right\|_{k}\right\} \\
& \quad \leqslant\left(\frac{2 \rho^{\prime} k \mathrm{e}^{2}}{3+2 N}\right)^{N+3 / 2} \exp \left\{-\frac{1}{2}\left[\sqrt{2 \rho^{\prime} k}-\left\|\frac{\theta^{\prime}}{\varepsilon}\right\|_{k}\right]^{2}\right\} \\
& \quad \leqslant\left(\frac{2 \rho^{\prime} k \mathrm{e}^{2}}{3+2 N}\right)^{N+3 / 2} \exp \left\{-\rho^{\prime} k+\sqrt{2 \rho^{\prime} k}\left\|\frac{\theta^{\prime}}{\varepsilon}\right\|_{k}\right\} .
\end{aligned}
$$

In the rest of the proof we set

$$
M=2\left[\sqrt{\log \left[\log \left(\varepsilon^{-1}\right) \vee 1\right]}\right] .
$$

For $\varepsilon$ small enough this choice of $M$ satisfies the assumptions on $M$ imposed above, since $W^{*} \asymp \log (1 / \varepsilon)$. Since $M \rightarrow \infty$ as $\varepsilon \rightarrow 0$, for any small constant $c>0$ there exists $\varepsilon_{0}$ such that for $\varepsilon<\varepsilon_{0}$ we have $\exp [-\gamma(M-N)] \leqslant c\left(\rho^{\prime}-\rho\right)^{2}$. Then for $\varepsilon<\varepsilon_{0}$, in view of (54) we have $-\left(\rho^{\prime}-\rho\right) k+\sqrt{2 \rho^{\prime} k}\left\|\theta^{\prime} / \varepsilon\right\|_{k} \leqslant$ $-\left(\rho^{\prime}-\rho\right) k / 2$ and, therefore,

$$
\begin{aligned}
& \sup _{\theta \in \Theta(\alpha, L)} \sum_{k=W^{*}+M}^{\infty} \exp (\rho k) r_{k} \mathbf{E} \xi_{k}^{2} \mathbf{I}\left\{\left\|\theta^{\prime}+\varepsilon \xi\right\|_{k}^{2} \geqslant 2 \varepsilon^{2} \rho^{\prime} k\right\} \\
& \quad \leqslant \sup _{\theta \in \Theta(\alpha, L)} \sum_{k=W^{*}+M}^{\infty}\left(\frac{2 \rho^{\prime} k \mathrm{e}^{2}}{3+2 N}\right)^{N+3 / 2} r_{k} \exp \left\{-\left(\rho^{\prime}-\rho\right) k+\sqrt{2 \rho^{\prime} k}\left\|\frac{\theta^{\prime}}{\varepsilon}\right\|_{k}\right\}
\end{aligned}
$$




$$
\begin{aligned}
& \leqslant \sum_{k=W^{*}+M}^{\infty}\left(\frac{2 \rho^{\prime} k \mathrm{e}^{2}}{3+2 N}\right)^{N+3 / 2} r_{k} \exp \left\{-\left(\rho^{\prime}-\rho\right) \frac{k}{2}\right\} \\
& \leqslant C r_{W^{*}}\left(\frac{2 \rho^{\prime}\left(W^{*}+M\right) \mathrm{e}^{2}}{3+2 N}\right)^{N+3 / 2}\left(\rho^{\prime}-\rho\right)^{-1} \exp \left\{-\left(\rho^{\prime}-\rho\right) \frac{W^{*}}{2}\right\},
\end{aligned}
$$

where the last inequality follows from (33) of Lemma 2 with the subexponential sequence $w_{k}=k^{N+3 / 2} r_{k}$ and from Lemma 1 with $w_{k}=r_{k}$. This inequality and (52), (53) yield

$$
\begin{aligned}
S_{3} & \leqslant C\left[\exp (-\alpha M) \psi_{\varepsilon}(\alpha, L)+\left(\rho^{\prime}-\rho\right)^{-1} \varepsilon^{2} r_{W^{*}}\right] \\
& \leqslant C \psi_{\varepsilon}(\alpha, L)\left[\exp (-\alpha M)+\left(\left(\rho^{\prime}-\rho\right) W^{*}\right)^{-1}\right] .
\end{aligned}
$$

Combining this result with (37), (38), and (51) we find

$$
\begin{aligned}
\sup _{\theta \in \Theta(\alpha, L)} \frac{R^{\varepsilon}\left(\theta^{*}, \theta\right)}{\psi_{\varepsilon}(\alpha, L)} \leqslant & V(1+\delta)^{2} \\
+C[ & \exp (-\alpha M)+\exp (-\rho M)+\exp \left(-\frac{N}{2} \min (\alpha, \rho)\right) \\
& \left.+\frac{N+\exp (\rho M)+\left(\rho^{\prime}-\rho\right)^{-1}}{W^{*}}\right] .
\end{aligned}
$$

It remains to take limits as $\varepsilon \rightarrow 0$, and then as $\delta \rightarrow 0$, using the definition of $M$ in (56) and the definition of $V$. This completes the proof of (26).

$\mathrm{Pr}$ o of of $\mathrm{Th}$ e or e $\mathrm{m} 2$. We follow the lines of the proof of Theorem 1 with $M=2 N$ (cf. (56)). The argument preceding (56) is true for any fixed $N$ and $\rho^{\prime}>\rho$, and it remains intact. Inspection of the proof of Theorem 1 after (56) shows that the choice of $N$ and $\rho^{\prime}$ defined in Theorem 2 is sufficient to get (27).

4.2. Proof of Theorem 3. For an integer $M$, consider the set

$$
\Theta_{\infty}^{M}(\alpha, L)=\left\{\theta \in \Theta_{\infty}(\alpha, L): \theta_{k}=0, \text { for }|k|>M\right\}
$$

and define

$$
A_{M}(\alpha, L)=\sup _{\theta \in \Theta_{\infty}^{M}(\alpha, L)} \sum_{k=-M}^{M} \exp (\rho k) \theta_{k}^{2} .
$$

Lemma 4. For any $\alpha>0, L>0$,

$$
\lim _{M \rightarrow \infty} A_{M}(\alpha, L)=A^{*}(\alpha, L) .
$$

$\mathrm{Pr}$ o o f. It is sufficient to note that

$$
A_{M}(\alpha, L)=\sup _{\theta \in \Theta_{\infty}(\alpha, L)} \sum_{|k| \leqslant M} \exp (\rho k) \theta_{k}^{2}
$$


and that uniformly in $\theta \in \Theta_{\infty}(\alpha, L)$

$$
\begin{aligned}
& \sum_{k=-\infty}^{\infty} \exp (\rho k) \theta_{k}^{2}-\sum_{|k| \leqslant M} \exp (\rho k) \theta_{k}^{2}=\sum_{k<-M} \exp (\rho k) \theta_{k}^{2} \\
& +\sum_{k>M} \exp (-\alpha k) \exp (\gamma k) \theta_{k}^{2} \leqslant \exp (-\rho M)+\exp (-\alpha M) E^{*}(\alpha, L) .
\end{aligned}
$$

Lemma 5. Under the assumptions of Theorem 3, for any $0<\alpha_{0}<$ $\alpha^{\prime} \leqslant \alpha$ and any $L_{0}, L, L^{\prime}>0$, we have

$$
\liminf _{\varepsilon \rightarrow \infty} \inf _{\hat{\theta}} \max \left\{\sup _{\theta \in \Theta\left(\alpha_{0}, L_{0}\right)} \frac{R^{\varepsilon}(\hat{\theta}, \theta)}{\psi_{\varepsilon}\left(\alpha_{0}, L_{0}\right)}, \sup _{\theta \in \Theta(\alpha, L)} \frac{R^{\varepsilon}(\hat{\theta}, \theta)}{\psi_{\varepsilon}\left(\alpha^{\prime}, L^{\prime}\right)}\right\} \geqslant 1-\frac{\gamma_{0}}{\gamma^{\prime}}
$$

where $\inf _{\hat{\theta}}$ denotes the infimum over all estimators and $\gamma_{0}=\alpha_{0}+\rho, \gamma^{\prime}=$ $\alpha^{\prime}+\rho$.

P r o o f. We will write for brevity $W_{0}=W^{*}\left(\alpha_{0}, L_{0}\right), W^{\prime}=W^{*}\left(\alpha^{\prime}, L^{\prime}\right)$. Let $M$ be a given integer satisfying $1 \leqslant M<W_{0}$, and let $\delta \in(0,1)$. For $L_{\delta}=L_{0}(1-\delta)$ set

$$
\Theta_{0}^{M}=\left\{\theta: q_{W_{0}} \sum_{\left|k-W_{0}\right| \leqslant M} \exp \left(\alpha_{0} k\right) \theta_{k}^{2} \leqslant L_{\delta}, \theta_{k}=0,\left|k-W_{0}\right|>M\right\} .
$$

Since $W_{0} \rightarrow \infty$, as $\varepsilon \rightarrow 0$, Lemma 1 implies that $q_{W_{0}} / q_{W_{0}+k}=1+o(1)$ uniformly in $|k| \leqslant M$. Hence $\Theta_{0}^{M} \subset \Theta\left(\alpha_{0}, L_{0}\right)$ for sufficiently small $\varepsilon$, and therefore

$$
\begin{aligned}
\mathscr{R}_{\varepsilon} & \stackrel{\text { def }}{=} \inf _{\hat{\theta}} \max \left\{\sup _{\theta \in \Theta\left(\alpha_{0}, L_{0}\right)} \frac{R^{\varepsilon}(\hat{\theta}, \theta)}{\psi_{\varepsilon}\left(\alpha_{0}, L_{0}\right)}, \sup _{\theta \in \Theta(\alpha, L)} \frac{R^{\varepsilon}(\hat{\theta}, \theta)}{\psi_{\varepsilon}\left(\alpha^{\prime}, L^{\prime}\right)}\right\} \\
& \geqslant \sup _{\theta \in \Theta_{0}^{M}} \inf _{\hat{\theta}} \max \left\{\frac{R^{\varepsilon}(\hat{\theta}, \theta)}{\psi_{\varepsilon}\left(\alpha_{0}, L_{0}\right)}, \frac{R^{\varepsilon}(\hat{\theta}, \mathbf{0})}{\psi_{\varepsilon}\left(\alpha^{\prime}, \mathbf{L}^{\prime}\right)}\right\},
\end{aligned}
$$

where 0 denotes the sequence $\theta$ with all the elements equal to 0 . To handle the last expression, we use again a renormalization. Change the variables from $\theta_{k}$ to $\nu_{k}$ by setting, for $|k| \leqslant M$,

$$
\nu_{k}=\frac{\theta_{k+W_{0}}}{\varepsilon \sqrt{2 \rho W_{0} r_{W_{0}} \exp \left[\rho\left(k+W_{0}\right)\right]}},
$$

and let $\widehat{\nu}_{k}$ be obtained from $\hat{\theta}_{k}$ by the same transformation, thus defining a sequence $\widehat{\nu} \in \Xi$.

Clearly, $\theta \in \Theta_{0}^{M}$ if and only if $\nu=\left\{\nu_{k}\right\}$ belongs to the set

$$
\Xi_{0}^{M}=\left\{\nu \in \Xi: \sum_{|k| \leqslant M} \exp \left(\gamma_{0} k\right) \nu_{k}^{2} \leqslant E_{\delta}, \nu_{k}=0,|k|>M\right\},
$$

where $E_{\delta}=E^{*}\left(\alpha_{0}, L_{0}\right)(1-\delta)$. We will denote by $\mathbf{P}_{\nu}$ the Gaussian measure generated by the observations $Y_{k}=\nu_{k}+\xi_{k+W_{0}} / \sqrt{2 \rho W_{0}},|k| \leqslant M$, and by $\mathbf{E}_{\nu}$ the expectation with respect to this measure. 
Using this notation and noticing that, in view of Lemma $1, r_{W_{0}} / r_{W_{0}+k}=$ $1+o(1)$ uniformly in $|k| \leqslant M$, we obtain

$$
\begin{aligned}
\frac{R^{\varepsilon}(\hat{\theta}, \theta)}{\psi_{\varepsilon}\left(\alpha_{0}, L_{0}\right)} & =\frac{1+o(1)}{A^{*}\left(\alpha_{0}, L_{0}\right)}\left[\sum_{|k| \leqslant M} \exp (\rho k) \mathbf{E}_{\nu}\left(\widehat{\nu}_{k}-\nu_{k}\right)^{2}+\sum_{|k|>M} \exp (\rho k) \mathbf{E}_{\nu} \widehat{\nu}_{k}^{2}\right] \\
& \geqslant \frac{1-\delta}{A^{*}\left(\alpha_{0}, L_{0}\right)} \sum_{|k| \leqslant M} \exp (\rho k) \mathbf{E}_{\nu}\left(\widehat{\nu}_{k}-\nu_{k}\right)^{2}
\end{aligned}
$$

which implies, together with (59), that

$$
\begin{gathered}
\mathscr{R}_{\varepsilon} \geqslant \frac{(1-\delta)}{A^{*}\left(\alpha_{0}, L_{0}\right)} \sup _{\nu \in \Xi_{0}^{M}} \inf _{\hat{\nu}}\left[(1-\delta) \sum_{|k| \leqslant M} \exp (\rho k) \mathbf{E}_{\nu}\left(\widehat{\nu}_{k}-\nu_{k}\right)^{2}\right. \\
\left.+\delta \lambda_{\varepsilon} \sum_{|k| \leqslant M} \exp (\rho k) \mathbf{E}_{0} \widehat{\nu}_{k}^{2}\right]
\end{gathered}
$$

where

$$
\lambda_{\varepsilon}=\frac{2 \varepsilon^{2} \rho W_{0} r_{W_{0}} \exp \left(\rho W_{0}\right)}{\psi_{\varepsilon}\left(\alpha^{\prime}, L^{\prime}\right)}=\frac{A^{*}\left(\alpha_{0}, L_{0}\right) W_{0} r_{W_{0}} \exp \left(\rho W_{0}\right)}{A^{*}\left(\alpha_{1}, L^{\prime}\right) W^{\prime} r_{W^{\prime}} \exp \left(\rho W^{\prime}\right)} .
$$

Since $\nu_{k},|k| \leqslant M$, are assumed to be known, the Bayes estimator which minimizes the left-hand side of $(60)$ can be found easily:

$$
\begin{aligned}
\widehat{\nu}_{k} & =\nu_{k}\left[1+\frac{\delta \lambda_{\varepsilon}}{1-\delta} \exp \left(\rho W_{0} \sum_{|k| \leqslant M}\left(Y_{k}-\nu_{k}\right)^{2}-\rho W_{0} \sum_{|k| \leqslant M} Y_{k}^{2}\right)\right]^{-1} \\
& =\nu_{k}\left[1+\frac{\delta \lambda_{\varepsilon}}{1-\delta} \exp \left(-\sqrt{2 \rho W_{0}} \sum_{|k| \leqslant M} \xi_{k+W_{0}} \nu_{k}-\rho W_{0} \sum_{|k| \leqslant M} \nu_{k}^{2}\right)\right]^{-1} .
\end{aligned}
$$

Next substituting this estimator in (60) one obtains

$$
\begin{aligned}
\mathscr{R}_{\varepsilon} \geqslant & \frac{(1-\delta)}{A^{*}\left(\alpha_{0}, L_{0}\right)} \sup _{\nu \in \Xi_{0}^{M}}\left[(1-\delta) \sum_{|k| \leqslant M} \exp (\rho k) \mathbf{E}_{\nu}\left(\widehat{\nu}_{k}-\nu_{k}\right)^{2}\right] \\
\geqslant & \frac{(1-\delta)^{2}}{A^{*}\left(\alpha_{0}, L_{0}\right)} \sup _{\nu \in \Xi_{0}^{M}}\left[\sum_{|k| \leqslant M} \exp (\rho k) \nu_{k}^{2}\right] \\
& \times \mathbf{E}\left\{1-\left[1+\frac{\delta \lambda_{\varepsilon}}{1-\delta} \exp \left(\sqrt{2 \rho W_{0}} \xi\|\nu\|-\rho W_{0}\|\nu\|^{2}\right)\right]^{-1}\right\}^{2},
\end{aligned}
$$

where $\xi \sim \mathscr{N}(0,1)$. In order to continue this inequality note that (see (12) and (61))

$$
\lim _{\varepsilon \rightarrow 0} \frac{\log \left(\lambda_{\varepsilon}\right)}{\rho W_{0}}=\lim _{\varepsilon \rightarrow 0} \frac{W_{0}-W^{\prime}}{W_{0}}=1-\frac{\gamma_{0}}{\gamma^{\prime}} .
$$

Then for all sufficiently small $\varepsilon \leqslant \varepsilon_{\delta}$ we have

$$
\mathbf{E}\left\{1-\left[1+\frac{\delta \lambda_{\varepsilon}}{1-\delta} \exp \left(\sqrt{2 \rho W_{0}} \xi\|\nu\|-\rho W_{0}\|\nu\|^{2}\right)\right]^{-1}\right\}^{2}
$$




$$
\begin{aligned}
& \geqslant(1-\delta)^{2} \mathbf{P}\left\{\frac{\delta^{2} \lambda_{\varepsilon}}{(1-\delta)^{2}} \exp \left(\sqrt{2 \rho W_{0}} \xi\|\nu\|-\rho W_{0}\|\nu\|^{2}\right)>1\right\} \\
& =(1-\delta)^{2} \mathbf{P}\left\{\xi \geqslant \sqrt{\frac{\rho W_{0}}{2}}\|\nu\|-\frac{1}{\sqrt{2 \rho W_{0}}\|\nu\|} \log \frac{\delta^{2} \lambda_{\varepsilon}}{(1-\delta)^{2}}\right\} \\
& \geqslant(1-\delta)^{3} \mathbf{I}\left\{\rho W_{0}\|\nu\|^{2}-\log \frac{\delta^{2} \lambda_{\varepsilon}}{(1-\delta)^{2}} \leqslant-2 \sqrt{-\rho W_{0} \log \delta}\|\nu\|\right\} \\
& =(1-\delta)^{3} \mathbf{I}\left\{\|\nu\|^{2} \leqslant \frac{1}{\rho W_{0}} \log \frac{\delta^{2} \lambda_{\varepsilon}}{(1-\delta)^{2}}-2 \sqrt{-\frac{\log \delta}{\rho W_{0}}}\|\nu\|\right\} \\
& \geqslant(1-\delta)^{3} \mathbf{I}\left\{\|\nu\|^{2} \leqslant 1-\frac{\gamma_{0}}{\gamma^{\prime}}-\delta\right\} .
\end{aligned}
$$

Combining this inequality with (62) one obtains

$$
\mathscr{R}_{\varepsilon} \geqslant \frac{(1-\delta)^{5}}{A^{*}\left(\alpha_{0}, L_{0}\right)} \sup _{\nu \in \Xi_{1}^{M}} \sum_{|k| \leqslant M} \exp (\rho k) \nu_{k}^{2}
$$

where

$$
\Xi_{1}^{M}=\left\{\nu \in \Xi_{0}^{M}:\|\nu\|^{2} \leqslant 1-\frac{\gamma_{0}}{\gamma^{\prime}}-\delta\right\} .
$$

Using the change of variables $\nu_{k}=\left[1-\gamma_{0} / \gamma^{\prime}-\delta\right]^{1 / 2} \nu_{k}^{\prime}$ we easily arrive at

$$
\sup _{\nu \in \Xi_{1}^{M}} \sum_{|k| \leqslant M} \exp (\rho k) \nu_{k}^{2} \geqslant\left(1-\frac{\gamma_{0}}{\gamma^{\prime}}-\delta\right) \sup _{\nu \in \Theta_{\infty}(\alpha, L)} \sum_{|k| \leqslant M} \exp (\rho k) \nu_{k}^{2},
$$

provided that $\left(1-\gamma_{0} / \gamma^{\prime}-\delta\right)^{-1}(1-\delta) \geqslant 1$.

To finish the proof of the lemma it remains to substitute (64) in (63) and to take the limits of the resulting inequality first as $\varepsilon \rightarrow 0$ then as $M \rightarrow \infty$ (using Lemma 4), and finally as $\delta \rightarrow 0$.

Lemma 6. Let $s_{\varepsilon}(\alpha, L)$ be defined by (15)-(16) and let $r_{k}$ and $q_{k}$ be sub-exponential. Then

$$
\liminf _{\varepsilon \rightarrow \infty} \inf _{\hat{\theta}} \sup _{\theta \in \Theta(\alpha, L)} \frac{R^{\varepsilon}(\hat{\theta}, \theta)}{s_{\varepsilon}(\alpha, L)}>0
$$

where $\inf _{\hat{\theta}}$ denotes the infimum over all estimators.

P r o o f. Consider the following parametric family in $\Theta(\alpha, L)$. Assume that all components $\theta_{k}$ are zero except $\theta_{W(\alpha, L)}=\varepsilon \sqrt{r_{W(\alpha, L)} \exp [\rho W(\alpha, L)]} \nu$, where $W(\alpha, L)$ is defined by (15) and $\nu$ is an unknown parameter such that

$$
\nu^{2} \leqslant \frac{L \exp [-\gamma W(\alpha, L)]}{\varepsilon^{2} r_{W(\alpha, L)} q_{W(\alpha, L)}}=c_{\varepsilon}(\alpha, L) .
$$

Using (15) and the facts that $W(\alpha, L) \rightarrow \infty$, as $\varepsilon \rightarrow 0$, and the sequences $r_{k}$ and $q_{k}$ are sub-exponential, we get that for $\varepsilon$ small enough $c_{\varepsilon}(\alpha, L) \geqslant c_{0}$ with 
some constant $c_{0}>0$. Therefore we have

$$
\begin{aligned}
\inf _{\hat{\theta}} \sup _{\theta \in \Theta(\alpha, L)} R^{\varepsilon}(\hat{\theta}, \theta) & \geqslant \varepsilon^{2} r_{W(\alpha, L)} \exp [\rho W(\alpha, L)] \inf _{\hat{\nu}} \sup _{|\nu| \leqslant \sqrt{c_{0}}} \mathbf{E}_{\nu}[\hat{\nu}(Z)-\nu]^{2} \\
& \geqslant C \varepsilon^{2} r_{W(\alpha, L)} \exp [\rho W(\alpha, L)]=C s_{\varepsilon}(\alpha, L),
\end{aligned}
$$

where $\mathbf{E}_{\nu}$ is the expectation with respect to distribution of a single Gaussian observation

$$
Z=\frac{Y_{W(\alpha, L)}}{\varepsilon \sqrt{r_{W(\alpha, L)} \exp [\rho W(\alpha, L)]}}=\nu+\xi_{W(\alpha, L)}
$$

and $\inf _{\hat{\nu}}$ denotes the infimum over all Borel functions $\hat{\nu}$.

$\mathrm{Pr}$ o of of $\mathrm{Th}$ e or e $\mathrm{m}$. The assumption of the theorem guarantees that there exists $\delta \in(0,1)$ such that

$$
\sup _{\theta \in \Theta\left(\alpha_{0}, L_{0}\right)} \frac{R^{\varepsilon}(\hat{\theta}, \theta)}{\psi_{\varepsilon}\left(\alpha_{0}, L_{0}\right)} \leqslant 1-\delta
$$

for all $\varepsilon$ small enough. Substituting this into (58) and choosing in (58) the value $\alpha^{\prime}=4 \gamma_{0} / \delta-\rho>\alpha_{0}$ we get for $\alpha>\alpha^{\prime}$ and for sufficiently small $\varepsilon$

$$
\inf _{\hat{\theta}} \max \left\{1-\delta, \sup _{\theta \in \Theta(\alpha, L)} \frac{R^{\varepsilon}(\hat{\theta}, \theta)}{\psi_{\varepsilon}\left(\alpha^{\prime}, L^{\prime}\right)}\right\} \geqslant 1-\frac{\delta}{4}+o(1) \geqslant 1-\frac{\delta}{2} .
$$

Thus, for $\varepsilon$ small enough,

$$
\begin{aligned}
\sup _{\theta \in \Theta(\alpha, L)} \frac{R^{\varepsilon}(\hat{\theta}, \theta)}{\psi_{\varepsilon}(\alpha, L)} & \geqslant\left(1-\frac{\delta}{2}\right) \frac{\psi_{\varepsilon}\left(\alpha^{\prime}, L^{\prime}\right)}{\psi_{\varepsilon}(\alpha, L)} \\
& =\left(1-\frac{\delta}{2}\right) \frac{A^{*}\left(\alpha^{\prime}, L^{\prime}\right) W^{\prime} r_{W^{\prime}} \exp \left(\rho W^{\prime}\right)}{A^{*}(\alpha, L) W r_{W} \exp (\rho W)}
\end{aligned}
$$

where $W$ and $W^{\prime}$ are defined by

$$
\begin{aligned}
W & =W^{*}(\alpha, L)=\frac{1+o(1)}{\rho+\alpha} \log \frac{L}{\varepsilon^{2}}, \\
W^{\prime} & =W^{*}\left(\alpha^{\prime}, L^{\prime}\right)=\frac{1+o(1)}{\rho+\alpha^{\prime}} \log \frac{L^{\prime}}{\varepsilon^{2}}, \quad \varepsilon \rightarrow 0 .
\end{aligned}
$$

On the other hand, it follows from Lemma 6 that the minimax risk for $\Theta\left(\alpha_{0}, L_{0}\right)$ satisfies

$$
\begin{aligned}
\inf _{\hat{\theta}} \sup _{\theta \in \Theta\left(\alpha_{0}, L_{0}\right)} \frac{R^{\varepsilon}(\hat{\theta}, \theta)}{\psi_{\varepsilon}\left(\alpha_{0}, L_{0}\right)} & \geqslant \frac{C r_{W\left(\alpha_{0}, L_{0}\right)} \exp \left[\rho W\left(\alpha_{0}, L_{0}\right)\right]}{W^{*}\left(\alpha_{0}, L_{0}\right) r_{W^{*}\left(\alpha_{0}, L_{0}\right)} \exp \left[\rho W^{*}\left(\alpha_{0}, L_{0}\right)\right]} \\
& \geqslant \exp [o(1) W] .
\end{aligned}
$$

In the last line we used that $W\left(\alpha_{0}, L_{0}\right)=(1+o(1)) W^{*}\left(\alpha_{0}, L_{0}\right)$ and $W^{*}\left(\alpha_{0}, L_{0}\right)=O(W)$, as $\varepsilon \rightarrow 0$. Finally, using (65)-(67), we get $\sup _{\theta \in \Theta\left(\alpha_{0}, L_{0}\right)} \frac{R^{\varepsilon}(\hat{\theta}, \theta)}{\psi_{\varepsilon}\left(\alpha_{0}, L_{0}\right)} \sup _{\theta \in \Theta(\alpha, L)} \frac{R^{\varepsilon}(\hat{\theta}, \theta)}{\psi_{\varepsilon}(\alpha, L)} \geqslant \frac{\exp [o(1) W] W^{\prime} r_{W^{\prime}} \exp \left(\rho W^{\prime}\right)}{W r_{W} \exp (\rho W)} \longrightarrow \infty$ as $\varepsilon \rightarrow 0$. 


\section{REFERENCES}

1. Бакушинский $А . Б$ Б. О построении регуляризующих алгоритмов при случайных помехах. - Докл. АН СССР, 1969, т. 189, № 2, с. 231-233.

2. Cavalier L., Golubev G.K., Picard D., Tsybakov A. B. Oracle inequalities for inverse problems. - Ann. Statist., 2002, v. 30, № 3, p. 843-874.

3. Cavalier L., Tsybakov A.B. Sharp adaptation for inverse problems with random noise. - Probab. Theory Relat. Fields, 2002, v. 123, № 3, p. 323-354.

4. Efromovich $S$. Robust and efficient recovery of a signal passed through a filter and then contaminated by nonGaussian noise. - IEEE Trans. Inf. Theory, 1997, v. 43, № 4, p. 1184-1191.

5. Efromovich $S$., Koltchinskii $V$. On inverse problems with unknown operators. - IEEE Trans. Inform. Theory, 2001, v. 47, № 7, p. 2876-2893.

6. Ermakov M.S. Minimax estimation of the solution of an ill-posed convolution type problem. - Проблемы передачи информации, 1989, т. 25, № 3, p. 28-39.

7. Goldenshluger A., Pereverzev S.V. Adaptive estimation of linear functionals in Hilbert scales from indirect white noise observations. - Probab. Theory Relat. Fields, 2000 , v. 118, № 2, p. 169-186.

8. Голубев Г. К., Хасьминский Р. З. Статистический подход к некоторым обратным задачам для уравнений в частных производных. - Проблемы передачи информации, 1999 , т. 35 , в. 2 , с. $51-66$.

9. Golubev G. K., Khasminskii R.Z. A statistical approach to the Cauchy problem for the Laplace equation. - State of the Art in Probability and Statistics. Festschrift for Willem R. van Zwet. Ed. by M. de Gunst, C. Klaassen, A. van der Vaart, Beachwood, OH: IMS, Institute of Mathematical Statistics, 2000, p. 419-433. (IMS Lecture notes - Monograph Series, v. 36.)

10. Hall P., Kerkyacharian G., Picard D. Block threshold rules for curve estimation using kernel and wavelet methods. - Ann. Statist., 1998, v. 26, № 3, p. 922-942.

11. Johnstone I. M. Wavelet shrinkage for correlated data and inverse problems: Adaptivity results. - Statistica Sinica, 1999, v. 9, № 1, p. 51-83.

12. Лепский О.В. Об одной задаче адаптивнот оценивания в гауссовском белом шуме. - Теория вероятн. и ее примен., 1990 , т. 35 , в. 3, с. 459-470.

13. Mair B., Ruymgaart F. H. Statistical inverse estimation in Hilbert scales. - SIAM J. Appl. Math., 1996, v. 56, № 5, p. 1424-1444.

14. Mathé P., Pereverzev S. V. Optimal discretization and degrees of ill-posedness for inverse estimation in Hilbert scales in the presence of random noise. Preprint 469, WIAS, Berlin.

15. Natterer $F$. Error bounds for Tikhonov regularization in Hilbert scales. - Appl. Anal., 1984 , v. 18 , p. $29-37$.

16. Pensky M., Vidakovic B. Adaptive wavelet estimator for nonparametric density deconvolution. - Ann. Statist., 1999, v. 27, № 6, p. 2033-2053.

17. Судажов B. Н., Халфин Л. А. Статистический подход к корректности задач математической физики. - Докл. АН СССР, 1964, т. 157, № 5, с. 1058-1060.

18. Tsybakov A.B. Pointwise and sup-norm sharp adaptive estimation of functions on the Sobolev classes. - Ann. Statist., 1998, v. 26, p. 2420-2469.

19. Tsybakov A.B. On the best rate of adaptive estimation in some inverse problems. C. R. Acad. Sci. Paris, Math. Ser. I, 2000, v. 330, p. 835-840.

Поступила в редакцию 23.VII.2002 